\title{
CAUCHY-TYPE MEANS FOR POSITIVE LINEAR FUNCTIONALS
}

\author{
M. ANWAR ${ }^{1}$, J. PEČARIĆ ${ }^{2}$ and M. RODIĆ LIPANOVIĆ ${ }^{3}$
}

\begin{abstract}
Some mean-value theorems of the Cauchy type, which are connected with Jensen's inequality, are given in [8] in discrete form and in [11] in integral form. Here we give the generalization of that result for positive linear functionals. Using that result, new means of Cauchy type for positive linear functionals are given. Monotonicity of these new means is also discussed.
\end{abstract}

\section{Introduction}

The well-known Jensen inequality asserts that for function $f$ holds

$$
f\left(\frac{1}{P_{n}} \sum_{i=1}^{n} p_{i} x_{i}\right) \leq \frac{1}{P_{n}} \sum_{i=1}^{n} p_{i} f\left(x_{i}\right)
$$

if and only if $f$ is convex function on interval $I \subseteq \mathbb{R}$, where $p_{i}$ are positive real numbers and $x_{i} \in I(i=1, \ldots, n)$, while $P_{n}=\sum_{i=1}^{n} p_{i}$.

In [10, p.9] authors gave an estimation of the quotient of differences of the left and the right side of the Jensen inequality for different functions, assuming that $p_{i}$ and $x_{i}$ are as above and $P_{n}=1$. Their result is a discrete version of a result previously given in [1].

Theorem 1.1. [10, p.9] Let $p_{i}>0(i=1, \ldots, n)$ with $P_{n}=1$, and $x_{i} \in I(i=1, \ldots, n)$ are not all equal. Let $f, g: I \rightarrow \mathbb{R}$ be twice differentiable functions such that

$$
0 \leq m \leq f^{\prime \prime}(x) \leq M \quad \text { and } \quad 0<k \leq g^{\prime \prime}(x) \leq K \quad \text { for all } x \in I .
$$

Then

$$
\frac{m}{K} \leq \frac{\sum_{i=1}^{n} p_{i} f\left(x_{i}\right)-f\left(\sum_{i=1}^{n} p_{i} x_{i}\right)}{\sum_{i=1}^{n} p_{i} g\left(x_{i}\right)-g\left(\sum_{i=1}^{n} p_{i} x_{i}\right)} \leq \frac{M}{k} .
$$

Corresponding author: Matloob Anwar.

2010 Mathematics Subject Classification. 26A24, 26E60.

Key words and phrases. Jensen's inequality, mean-value theorems, linear functional, generalized means, generalized power mean, Cauchy-type means.

The research of the second and the third author is supported by the Croatian Ministry of Science, Education and Sports under the Research Grant 117-1170889-0888. 
Under the same conditions on $p_{i}$ and $x_{i}, \mathrm{~A}$. McD. Mercer in [7] and [8] gave the following two mean-value theorems of the Lagrange and Cauchy type.

Theorem 1.2. Let I be a compact real interval and $f, g: I \rightarrow \mathbb{R}$. Let $x_{i} \in I$ and $p_{i}>0(i=1, \ldots, n)$ such that $P_{n}=1$.

(i) If $f \in C^{2}(I)$, then

$$
\sum_{i=1}^{n} p_{i} f\left(x_{i}\right)-f\left(\sum_{i=1}^{n} p_{i} x_{i}\right)=\frac{1}{2} f^{\prime \prime}(\xi)\left(\sum_{i=1}^{n} p_{i} x_{i}^{2}-\left(\sum_{i=1}^{n} p_{i} x_{i}\right)^{2}\right)
$$

holds for some $\xi \in I$.

(ii) If $f, g \in C^{2}(I)$, then

$$
\frac{\sum_{i=1}^{n} p_{i} f\left(x_{i}\right)-f\left(\sum_{i=1}^{n} p_{i} x_{i}\right)}{\sum_{i=1}^{n} p_{i} g\left(x_{i}\right)-g\left(\sum_{i=1}^{n} p_{i} x_{i}\right)}=\frac{f^{\prime \prime}(\xi)}{g^{\prime \prime}(\xi)}
$$

holds for some $\xi \in I$, provided that the denominator of the left-hand side is non-zero.

Remark 1.3. We use $f \in C^{2}(I)$ to denote that function $f$ has continuous second derivative on $I$.

Furthermore, having in mind the integral Jensen inequality, the authors in [11] gave similar results in integral form.

Theorem 1.4. ([11]) Let I be a compact real interval and $\varphi, \psi: I \rightarrow \mathbb{R}$. Let $h$ be an integrable function with respect to a normalized weight $\omega$ on $[a, b] \subset \mathbb{R}$ such that the range of $h$ is a subset of $I$.

(i) If $\varphi \in C^{2}(I)$, then

$$
\begin{aligned}
\int_{a}^{b} \varphi(h(x)) \omega(x) d x & -\varphi\left(\int_{a}^{b} h(x) \omega(x) d x\right) \\
& =\frac{1}{2} \varphi^{\prime \prime}(\xi)\left[\int_{a}^{b}(h(x))^{2} \omega(x) d x-\left(\int_{a}^{b} h(x) \omega(x) d x\right)^{2}\right]
\end{aligned}
$$

holds for some $\xi \in I$.

(ii) If $\varphi, \psi \in C^{2}(I)$, then

$$
\frac{\int_{a}^{b} \varphi(h(x)) \omega(x) d x-\varphi\left(\int_{a}^{b} h(x) \omega(x) d x\right)}{\int_{a}^{b} \psi(h(x)) \omega(x) d x-\psi\left(\int_{a}^{b} h(x) \omega(x) d x\right)}=\frac{\varphi^{\prime \prime}(\xi)}{\psi^{\prime \prime}(\xi)},
$$

holds for some $\xi \in I$, provided that the denominator of the left-hand side is non-zero. 
In [13] the generalization of these results is given for the case when the function $h$ is defined on a convex set $\Omega \subseteq \mathbb{R}^{n}$ equipped with a probability measure.

The aim of our paper here is to give further generalization of (5) and (6), for positive linear functionals, and also to define adequate Cauchy-type means.

\section{Mean-value theorems for positive linear functionals}

Let $E$ be a nonempty set and $L$ be a linear class of real-valued functions $f: E \rightarrow \mathbb{R}$ having the properties:

(L1) $f, g \in L \Rightarrow(a f+b g) \in L$ for all $a, b \in \mathbb{R}$

(L2) $1 \in L$, that is if $f(t)=1$ for $t \in E$, then $f \in L$.

A positive (isotonic) linear functional is a functional $A: L \rightarrow \mathbb{R}$ having the properties:

(A1) $A(a f+b g)=a A(f)+b A(g)$ for $f, g \in L, a, b \in \mathbb{R}(A$ is linear $)$;

(A2) $f \in L, f(t) \geq 0$ on $E \Rightarrow A(f) \geq 0$ ( $A$ is positive or isotonic).

B. Jessen in [6] (see also [12, p.47]) gave the following generalization of Jensen's inequality for positive linear functionals.

Theorem 2.1. Let $L$ satisfy properties $L 1, L 2$ on a nonempty set $E$ and let $\varphi: I \rightarrow \mathbb{R}$ be a continuous convex function on an interval $I \subset \mathbb{R}$. If $A$ is a positive linear functional on $L$ with $A(1)=1$, then for all $g \in L$ such that $\varphi(g) \in L$ we have $A(g) \in I$ and

$$
\varphi(A(g)) \leq A(\varphi(g))
$$

Using this result we shall give two mean-value theorems for positive linear functionals.

The following result (see for example [12, p.4]) will be very useful.

Lemma 2.2. Let $\varphi: I \rightarrow \mathbb{R}, I \subset \mathbb{R}$, be such that $\varphi \in C^{2}(I), \varphi^{\prime \prime}$ is bounded and $m=\inf _{t \in I} \varphi^{\prime \prime}(t)$, $M=\sup _{t \in I} \varphi^{\prime \prime}(t)$.

Then the functions $\varphi_{1}, \varphi_{2}: I \rightarrow \mathbb{R}$ defined by

$$
\begin{aligned}
\varphi_{1}(t) & =\frac{M}{2} t^{2}-\varphi(t) \\
\varphi_{2}(t) & =\varphi(t)-\frac{m}{2} t^{2}
\end{aligned}
$$

are convex functions. 
Now we give our mean-value theorems for positive linear functionals.

Theorem 2.3. Let $L$ satisfy properties $L 1, L 2$ on a nonempty set $E$ and let $\varphi: I \rightarrow \mathbb{R}, \varphi \in C^{2}(I)$, where $I \subset \mathbb{R}$ is a compact real interval. If $A$ is a positive linear functional on $L$ with $A(1)=1$, then for all $g \in L$ such that $g^{2}, \varphi(g) \in L$ there exists some $\xi \in I$ such that the following holds

$$
A(\varphi(g))-\varphi(A(g))=\frac{\varphi^{\prime \prime}(\xi)}{2}\left[A\left(g^{2}\right)-(A(g))^{2}\right] .
$$

Proof. Denote $m=\min _{t \in I} \varphi^{\prime \prime}(t), M=\max _{t \in I} \varphi^{\prime \prime}(t)$. The previous Lemma states that then the functions $\varphi_{1}, \varphi_{2}: I \rightarrow \mathbb{R}$ defined by (7) are convex functions. As they are also continuous, we can apply Theorem 2.1 on them.

We get

$$
A(\varphi(g))-\varphi(A(g)) \leq \frac{M}{2}\left[A\left(g^{2}\right)-(A(g))^{2}\right]
$$

and

$$
A(\varphi(g))-\varphi(A(g)) \geq \frac{m}{2}\left[A\left(g^{2}\right)-(A(g))^{2}\right] .
$$

Now combining these two inequalities and since $\varphi^{\prime \prime}$ is continuous, there exists some $\xi \in I$ $\left(m \leq \varphi^{\prime \prime}(\xi) \leq M\right)$ such that

$$
A(\varphi(g))-\varphi(A(g))=\frac{\varphi^{\prime \prime}(\xi)}{2}\left[A\left(g^{2}\right)-(A(g))^{2}\right] .
$$

Theorem 2.4. Let $L$ satisfy properties $L 1, L 2$ on a nonempty set $E$ and let $\varphi, \psi: I \rightarrow \mathbb{R}, \varphi, \psi \in$ $C^{2}(I)$, where $I \subset \mathbb{R}$ is a compact real interval. If $A$ is a positive linear functional on $L$ with $A(1)=1$, then for all $g \in L$ such that $g^{2}, \varphi(g), \psi(g) \in L$ and $A\left(g^{2}\right)-(A(g))^{2} \neq 0$, there exists some $\xi \in I$ such that the following holds

$$
\frac{A(\varphi(g))-\varphi(A(g))}{A(\psi(g))-\psi(A(g))}=\frac{\varphi^{\prime \prime}(\xi)}{\psi^{\prime \prime}(\xi)}
$$

provided that the denominator of the left-hand side is non-zero.

Proof. Consider the function $\chi$ defined by

$$
\chi(t)=[A(\psi(g))-\psi(A(g))] \cdot \varphi(t)-[A(\varphi(g))-\varphi(A(g))] \cdot \psi(t) .
$$

Function $\chi$ is linear combination of functions $\varphi$ and $\psi$, so $\chi \in C^{2}(I)$ and $\chi(g) \in L$ for all $g \in L$. Now we can apply Theorem 2.3 on function $\chi$ and it follows that there exists some $\xi \in I$ such that the following holds

$$
A(\chi(g))-\chi(A(g))=\frac{\chi^{\prime \prime}(\xi)}{2}\left[A\left(g^{2}\right)-(A(g))^{2}\right] .
$$


The left-hand side of this equation equals to zero, the term in the square brackets on the right-hand side is non-zero, so we have that

$$
\chi^{\prime \prime}(\xi)=0
$$

Now the assertion of our theorem follows directly.

\section{Cauchy-type means for positive linear functionals}

Let $L$ satisfy properties $L 1, L 2$ on a nonempty set $E$. Let $A$ be a positive linear functional (i.e. $A$ satisfies the conditions $A 1, A 2)$ on $L$ with $A(1)=1$, and let $g \in L$.

Then for a strictly monotone continuous function $\alpha$ such that $\alpha \circ g \in L$, the generalized quasi-arithmetic mean, $M_{\alpha}(g, A)$, of $g$ with respect to the positive linear functional $A$ and the function $\alpha$ is defined by (see for example [12, p.107])

$$
M_{\alpha}(g, A)=\alpha^{-1}(A(\alpha \circ g)) .
$$

The following theorem holds.

Theorem 3.1. Let $g \in L$ be such that the image of $g$ is a compact real interval $I$, and let $\alpha, \beta, \gamma$ : $I \rightarrow \mathbb{R}$ be strictly monotone functions, $\alpha, \beta, \gamma \in C^{2}(I)$, such that $\alpha \circ g, \beta \circ g, \gamma \circ g,(\gamma \circ g)^{2} \in L$ and $A\left((\gamma \circ g)^{2}\right)-(A(\gamma \circ g))^{2} \neq 0$.

Then

$$
\frac{\alpha\left(M_{\alpha}(g, A)\right)-\alpha\left(M_{\gamma}(g, A)\right)}{\beta\left(M_{\beta}(g, A)\right)-\beta\left(M_{\gamma}(g, A)\right)}=\frac{\alpha^{\prime \prime}(\eta) \cdot \gamma^{\prime}(\eta)-\alpha^{\prime}(\eta) \cdot \gamma^{\prime \prime}(\eta)}{\beta^{\prime \prime}(\eta) \cdot \gamma^{\prime}(\eta)-\beta^{\prime}(\eta) \cdot \gamma^{\prime \prime}(\eta)}
$$

holds for some $\eta$ in the image of $g$, provided that the denominator of the left-hand side is nonzero.

Proof. If we apply Theorem 2.4 on the functions

$$
\varphi=\alpha \circ \gamma^{-1}, \psi=\beta \circ \gamma^{-1}, g=\gamma \circ g,
$$

we find that there exists some $\xi$ such that

$$
\frac{\alpha\left(M_{\alpha}(g, A)\right)-\alpha\left(M_{\gamma}(g, A)\right)}{\beta\left(M_{\beta}(g, A)\right)-\beta\left(M_{\gamma}(g, A)\right)}=\frac{\alpha^{\prime \prime}\left(\gamma^{-1}(\xi)\right) \cdot \gamma^{\prime}\left(\gamma^{-1}(\xi)\right)-\alpha^{\prime}\left(\gamma^{-1}(\xi)\right) \cdot \gamma^{\prime \prime}\left(\gamma^{-1}(\xi)\right)}{\beta^{\prime \prime}\left(\gamma^{-1}(\xi)\right) \cdot \gamma^{\prime}\left(\gamma^{-1}(\xi)\right)-\beta^{\prime}\left(\gamma^{-1}(\xi)\right) \cdot \gamma^{\prime \prime}\left(\gamma^{-1}(\xi)\right)}
$$

Thus, by setting $\gamma^{-1}(\xi)=\eta$, we find that there exists some $\eta$ in the image of $g$ such that

$$
\frac{\alpha\left(M_{\alpha}(g, A)\right)-\alpha\left(M_{\gamma}(g, A)\right)}{\beta\left(M_{\beta}(g, A)\right)-\beta\left(M_{\gamma}(g, A)\right)}=\frac{\alpha^{\prime \prime}(\eta) \cdot \gamma^{\prime}(\eta)-\alpha^{\prime}(\eta) \cdot \gamma^{\prime \prime}(\eta)}{\beta^{\prime \prime}(\eta) \cdot \gamma^{\prime}(\eta)-\beta^{\prime}(\eta) \cdot \gamma^{\prime \prime}(\eta)}
$$

provided that the denominator of the left-hand side is non-zero. 
Corollary 1. Let the conditions of Theorem 3.1 hold.

Let

$$
\chi(\eta)=\frac{\alpha^{\prime \prime}(\eta) \cdot \gamma^{\prime}(\eta)-\alpha^{\prime}(\eta) \cdot \gamma^{\prime \prime}(\eta)}{\beta^{\prime \prime}(\eta) \cdot \gamma^{\prime}(\eta)-\beta^{\prime}(\eta) \cdot \gamma^{\prime \prime}(\eta)}
$$

be invertible function.

Then

$$
\eta=\chi^{-1}\left(\frac{\alpha\left(M_{\alpha}(g, A)\right)-\alpha\left(M_{\gamma}(g, A)\right)}{\beta\left(M_{\beta}(g, A)\right)-\beta\left(M_{\gamma}(g, A)\right)}\right)
$$

is a mean, provided that the denominator of the term in the brackets is non-zero.

Proof. Since $\eta$ is in the image of $g$, it follows that

$$
\min _{t \in E} g(t) \leq \chi^{-1}\left(\frac{\alpha\left(M_{\alpha}(g, A)\right)-\alpha\left(M_{\gamma}(g, A)\right)}{\beta\left(M_{\beta}(g, A)\right)-\beta\left(M_{\gamma}(g, A)\right)}\right) \leq \max _{t \in E} g(t) .
$$

This shows that this is a mean.

Now, from the results given above, we can deduce the corresponding results for the generalized power mean, $M_{r}(g, A)$, of $g$ with respect to the positive linear functional $A$ which is defined for $r \in \mathbb{R}$ by (see for example [12, p.108])

$$
M_{r}(g, A)= \begin{cases}\left(A\left(g^{r}\right)\right)^{\frac{1}{r}}, & r \neq 0 \\ \exp (A(\log g)), & r=0\end{cases}
$$

where $g(t)>0$ for $t \in E$, $\log g \in L$ and $g^{r} \in L$ for $r \in \mathbb{R} \backslash\{0\}$.

Corollary 2. Let $g \in L$ be such that the image of $g$ is a compact real interval I. Let $r, l, s \in \mathbb{R} \backslash\{0\}$, $r \neq l, s ; l \neq s$, such that $g^{r}, g^{l}, g^{s}, g^{2 s} \in L$ and $A\left(g^{2 s}\right)-\left(A\left(g^{s}\right)\right)^{2} \neq 0$. Then

$$
\frac{M_{r}^{r}(g, A)-M_{s}^{r}(g, A)}{M_{l}^{l}(g, A)-M_{s}^{l}(g, A)}=\frac{r(r-s)}{l(l-s)} \cdot \eta^{r-l}
$$

holds for some $\eta$ in the image of $g$, provided that the denominator of the left-hand side is nonzero.

Proof. If we set

$$
\alpha(t)=t^{r}, \beta(t)=t^{l}, \gamma(t)=t^{s},
$$

in Theorem 3.1, we get the assertion (16).

Since $\eta$ is in the image of $g$, (16) suggests a new mean as it is

$$
\min _{t \in E} g(t) \leq\left(\frac{l(l-s)}{r(r-s)} \frac{M_{r}^{r}(g, A)-M_{s}^{r}(g, A)}{M_{l}^{l}(g, A)-M_{s}^{l}(g, A)}\right)^{\frac{1}{r-l}} \leq \max _{t \in E} g(t)
$$


for $r, l, s \in \mathbb{R} \backslash\{0\}, r \neq l, s ; l \neq s ; A\left(g^{2 s}\right)-\left(A\left(g^{s}\right)\right)^{2} \neq 0$.

From (16) it follows that we can define a new mean $M_{r, l}^{[s]}(g, A)$ as follows

$$
M_{r, l}^{[s]}(g, A)=\left(\frac{l(l-s)}{r(r-s)} \frac{M_{r}^{r}(g, A)-M_{s}^{r}(g, A)}{M_{l}^{l}(g, A)-M_{s}^{l}(g, A)}\right)^{\frac{1}{r-l}},
$$

for $r, l, s \in \mathbb{R} \backslash\{0\}, r \neq l, s ; l \neq s ; A\left(g^{2 s}\right)-\left(A\left(g^{s}\right)\right)^{2} \neq 0$.

Similarly, we can calculate some other cases for $r, l, s \in \mathbb{R}$ and we get the following definition of $M_{r, l}^{[s]}(g, A)$.

Definition 3.2. Let $L$ satisfy properties $L 1, L 2$ on a nonempty set $E$ and let $A$ be a positive linear functional on $L$ with $A(1)=1$. Let $r, l, s \in \mathbb{R}$ and let $g \in L$ be such that the image of $g$ is a compact real interval $I \subset \mathbb{R}^{+}$, and $g^{r}, g^{l}, g^{s}, g^{2 s}, \log g, \log ^{2} g \in L$ for $r, l, s \in \mathbb{R} \backslash\{0\}$.

Then we define the Cauchy-type mean $M_{r, l}^{[s]}(g, A)$ of $g$ with respect to the positive linear functional $A$ by

$$
M_{r, l}^{[s]}(g, A)=\left(\frac{l(l-s)}{r(r-s)} \cdot \frac{M_{r}^{r}(g, A)-M_{s}^{r}(g, A)}{M_{l}^{l}(g, A)-M_{s}^{l}(g, A)}\right)^{\frac{1}{r-l}},
$$

for $r, l, s \neq 0 ; r \neq l, s ; l \neq s ; A\left(g^{2 s}\right)-\left(A\left(g^{s}\right)\right)^{2} \neq 0$;

$$
M_{r, 0}^{[s]}(g, A)=M_{0, r}^{[s]}(g, A)=\left(-\frac{s}{r(r-s)} \cdot \frac{M_{r}^{r}(g, A)-M_{s}^{r}(g, A)}{\log \left(M_{0}(g, A)\right)-\log \left(M_{s}(g, A)\right)}\right)^{\frac{1}{r}},
$$

for $r, s \neq 0 ; r \neq s ; A\left(g^{2 s}\right)-\left(A\left(g^{s}\right)\right)^{2} \neq 0$;

$$
M_{r, l}^{[0]}(g, A)=\left(\frac{l^{2}}{r^{2}} \cdot \frac{M_{r}^{r}(g, A)-M_{0}^{r}(g, A)}{M_{l}^{l}(g, A)-M_{0}^{l}(g, A)}\right)^{\frac{1}{r-l}},
$$

for $r, l \neq 0 ; r \neq l ; A\left(\log ^{2} g\right)-(A(\log g))^{2} \neq 0$;

where we suppose that all expressions are well defined.

\section{Monotonicity of Cauchy-type means for positive linear functionals}

The following Lemma is valid.

Lemma 4.1. Define the function $\varphi_{s}: \mathbb{R}^{+} \rightarrow \mathbb{R}$ by

$$
\varphi_{s}(x)= \begin{cases}\frac{x^{s}}{s(s-1)}, & s \neq 0,1 \\ -\log x, & s=0 \\ x \log x, & s=1 .\end{cases}
$$

It is $\varphi_{s}^{\prime \prime}(x)=x^{s-2}$, so $\varphi_{s}$ is convex function. 
We shall use the following result from [2].

Theorem 4.2. ([2]) Let L satisfy properties L1, L2 on a nonempty set $E$ and let $A$ be a positive linear functional on $L$ with $A(1)=1$. Let a positive function $g \in L$ be such that $g^{r} \in L$ for $r \in J \backslash\{0,1\},(J \subset \mathbb{R}), \log g \in L$ if $r=0 \in J$ and $g \log g \in L$ if $r=1 \in J$.

Let us define

$$
\Lambda_{t}(g, A)=A\left(\varphi_{t}(g)\right)-\varphi_{t}(A(g))
$$

(i) Then for all $s, t \in J$ we have

$$
\Lambda_{\frac{s+t}{2}}^{2} \leq \Lambda_{s} \cdot \Lambda_{t}
$$

That is, $t \mapsto \Lambda_{t}$ is log-convex in Jensen's sense.

(ii) If $t \mapsto \Lambda_{t}$ is continuous on J, then it is also log-convex. That is, for $r<s<t(r, s, t \in J)$ we have that

$$
\left(\Lambda_{s}\right)^{t-r} \leq\left(\Lambda_{r}\right)^{t-s} \cdot\left(\Lambda_{t}\right)^{s-r}
$$

We can represent the function $\Lambda_{t}$ defined by (18), where the function $\varphi_{t}$ is defined by (17), using generalized power means defined by (15), as

$$
\Lambda_{t}(g, A)= \begin{cases}\frac{1}{t(t-1)}\left[M_{t}^{t}(g, A)-M_{1}^{t}(g, A)\right], & t \neq 0,1 \\ -\log M_{0}(g, A)+\log M_{1}(g, A), & t=0 \\ M_{1}(g \log g, A)-M_{1}(g, A) \cdot \log \left(M_{1}(g, A)\right), & t=1 .\end{cases}
$$

Note that, as it follows from the previous Theorem, that if so defined function $t \mapsto \Lambda_{t}$ is continuous on $J$, then it is also log-convex.

The following Lemma is also valid.

Lemma 4.3. Define the function $\psi_{s}: \mathbb{R} \rightarrow \mathbb{R}$ by

$$
\psi_{s}(x)= \begin{cases}\frac{1}{s^{2}} e^{s x}, & s \neq 0 \\ \frac{1}{2} x^{2}, & s=0 .\end{cases}
$$

It is $\psi_{s}^{\prime \prime}(x)=e^{s x}$, so $\psi_{s}$ is convex function.

Remark 4.4. The authors in [2] proved that Theorem 4.2 also holds if we define function $\Lambda_{t}$ by

$$
\Lambda_{t}(g, A)=A\left(\psi_{t}(g)\right)-\psi_{t}(A(g))
$$

where the function $\psi_{t}$ is defined by (20). 
Now we can also represent our newly defined function $\Lambda_{t}$, which is defined by (21) where the function $\psi_{t}$ is defined by (20), using generalized power means, as follows

$$
\Lambda_{t}= \begin{cases}\frac{1}{t^{2}}\left[M_{t}^{t}(g, A)-M_{0}^{t}(g, A)\right], & t \neq 0 \\ \frac{1}{2}\left[M_{2}^{2}(\log g, A)-M_{1}^{2}(\log g, A)\right], & t=0 .\end{cases}
$$

Also note that if so defined function $t \mapsto \Lambda_{t}$ is continuous on $J$, then it is also log-convex.

The following result gives us the useful property for log-convex functions.

Lemma 4.5. ([4]) Let $f: J \rightarrow \mathbb{R}^{+}(J \subset \mathbb{R})$ be log-convex function and $x_{1}, x_{2}, y_{1}, y_{2} \in J$ such that $x_{1} \leq y_{1}, x_{2} \leq y_{2}, x_{1} \neq x_{2}, y_{1} \neq y_{2}$. Then the following inequality holds

$$
\left(\frac{f\left(x_{2}\right)}{f\left(x_{1}\right)}\right)^{\frac{1}{x_{2}-x_{1}}} \leq\left(\frac{f\left(y_{2}\right)}{f\left(y_{1}\right)}\right)^{\frac{1}{y_{2}-y_{1}}} .
$$

Now in the next theorem we give the comparison for our newly defined Cauchy-type means, $M_{r, l}^{[s]}$, from the previous section.

We shall suppose that $g$ and $A$ are such that both defined functions $\Lambda_{t}$ (in (18) and in (21)) are continuous functions.

Theorem 4.6. Let $a, b, c, d, s \in \mathbb{R}$ be such that $a \leq c, b \leq d, a \neq b, c \neq d$ and $s \neq a, b, c, d$.

Then, for $M_{r, l}^{[s]}$ defined in Definition 3.2 we have that

$$
M_{b, a}^{[s]} \leq M_{d, c}^{[s]}
$$

Proof. Case I.:

Let us consider the function $\Lambda_{t}$ defined by (18). As $\Lambda_{t}$ is log-convex, previous Lemma implies that for $a, b, c, d \in \mathbb{R}$ such that $a \leq c, b \leq d, a \neq b, c \neq d$, the following is valid

$$
\left(\frac{\Lambda_{b}}{\Lambda_{a}}\right)^{\frac{1}{b-a}} \leq\left(\frac{\Lambda_{d}}{\Lambda_{c}}\right)^{\frac{1}{d-c}}
$$

For $s>0$, by substituting: $g=f^{s}, a=\frac{a}{s}, b=\frac{b}{s}, c=\frac{c}{s}, d=\frac{d}{s}$, such that $\frac{a}{s} \leq \frac{c}{s}, \frac{b}{s} \leq \frac{d}{s}, a \neq b$, $c \neq d$, in $\Lambda_{b}$, we get

$$
\Lambda_{b, s}(f, A)= \begin{cases}\frac{s^{2}}{b(b-s)}\left[M_{b}^{b}(f, A)-M_{s}^{b}(f, A)\right], & b \neq 0, s \\ s\left[-\log M_{0}(f, A)+\log M_{s}(f, A)\right], & b=0 \\ s\left[M_{1}\left(f^{s} \log f, A\right)-M_{s}^{s}(f, A) \cdot \log \left(M_{s}(f, A)\right)\right], & b=s,\end{cases}
$$


and (25) becomes

$$
\left(\frac{\Lambda_{b, s}}{\Lambda_{a, s}}\right)^{\frac{s}{b-a}} \leq\left(\frac{\Lambda_{d, s}}{\Lambda_{c, s}}\right)^{\frac{s}{d-c}}
$$

As it is $s>0$, the statement (24) follows directly.

For $s<0$, by substituting: $g=f^{s}, a=\frac{a}{s}, b=\frac{b}{s}, c=\frac{c}{s}, d=\frac{d}{s}$, such that $\frac{a}{s} \geq \frac{c}{s}, \frac{b}{s} \geq \frac{d}{s}, a \neq b$, $c \neq d$, in $\Lambda_{b}$, we get again (26), but (25) becomes

$$
\left(\frac{\Lambda_{b, s}}{\Lambda_{a, s}}\right)^{\frac{s}{b-a}} \leq\left(\frac{\Lambda_{d, s}}{\Lambda_{c, s}}\right)^{\frac{s}{d-c}}
$$

Because it is $s<0$, we have that

$$
\left(\frac{\Lambda_{b, s}}{\Lambda_{a, s}}\right)^{\frac{1}{b-a}} \geq\left(\frac{\Lambda_{d, s}}{\Lambda_{c, s}}\right)^{\frac{1}{d-c}}
$$

where $a \geq c, b \geq d, a \neq b, c \neq d$ and we get our required result.

Case II.:

Let us consider the function $\Lambda_{t}$ defined by (21). As $\Lambda_{t}$ is log-convex, previous Lemma implies that for $a, b, c, d \in \mathbb{R}$ such that $a \leq c, b \leq d, a \neq b, c \neq d$, inequality (25) holds.

Therefore we have for $a, b, c, d \in \mathbb{R}$ such that $a \leq c, b \leq d, a \neq b, c \neq d$, that it holds

$$
M_{b, a}^{[0]} \leq M_{d, c}^{[0]} .
$$

Remark 4.7. If we set the functional $A$ of the function $g$ to be

$$
A(g)=\int_{\Omega} g(u) d \mu(u)
$$

where $\Omega \subseteq \mathbb{R}^{n}$ is a convex set equipped with a probability measure $\mu$, we get the means of Cauchy-type given in [4] and also adequate results.

\section{Some related results}

In this section we will give related results of Mercer's and Aczél's type.

\subsection{Means of the Mercer type}

In [5] the following version of Jessen's inequality is given. 
Theorem 5.2. Let $L$ satisfy properties $L 1, L 2$ on a nonempty set $E$ and let $\varphi: I \rightarrow \mathbb{R}$ be a continuous convex function on an interval $I=[m, M](-\infty<m<M<\infty)$. If $A$ is a positive linear functional on $L$ with $A(1)=1$, then for all $g \in L$ such that $\varphi(g), \varphi(m+M-g) \in L$ (so that $m \leq g(t) \leq M$ for all $t \in E$ ), we have the following variant of Jessen's inequality

$$
\varphi(m+M-A(g)) \leq \varphi(m)+\varphi(M)-A(\varphi(g)) .
$$

Using this result we shall give two mean-value theorems for positive linear functionals.

Theorem 5.3. Let $L$ satisfy properties $L 1, L 2$ on a nonempty set $E$ and let $\varphi: I \rightarrow \mathbb{R}, \varphi \in C^{2}(I)$, where $I=[m, M](-\infty<m<M<\infty)$. If $A$ is a positive linear functional on $L$ with $A(1)=1$, then for all $g \in L$ such that $g^{2}, \varphi(g), \varphi(m+M-g) \in L$ (so that $m \leq g(t) \leq M$ for all $t \in E$ ) there exists some $\xi \in I$ such that the following holds

$$
\begin{aligned}
\varphi(m)+\varphi(M)-A(\varphi(g))-\varphi( & m+M-A(g)) \\
= & \frac{\varphi^{\prime \prime}(\xi)}{2}\left[m^{2}+M^{2}-A\left(g^{2}\right)-(m+M-A(g))^{2}\right] .
\end{aligned}
$$

Proof. The proof is similar to the proof of Theorem 2.3 using Theorem 5.2 instead of Theorem 2.1 .

Theorem 5.4. Let $L$ satisfy properties $L 1, L 2$ on a nonempty set $E$ and let $\varphi, \psi: I \rightarrow \mathbb{R}, \varphi, \psi \in$ $C^{2}(I)$, where $I=[m, M](-\infty<m<M<\infty)$. If $A$ is a positive linear functional on $L$ with $A(1)=1$, then for all $g \in L$ such that $g^{2}, \varphi(g), \varphi(m+M-g), \psi(g), \psi(m+M-g) \in L$ (so that $m \leq g(t) \leq M$ for all $t \in E)$ and $m^{2}+M^{2}-A\left(g^{2}\right)-(m+M-A(g))^{2} \neq 0$, there exists some $\xi \in I$ such that the following holds

$$
\frac{\varphi(m)+\varphi(M)-A(\varphi(g))-\varphi(m+M-A(g))}{\psi(m)+\psi(M)-A(\psi(g))-\psi(m+M-A(g))}=\frac{\varphi^{\prime \prime}(\xi)}{\psi^{\prime \prime}(\xi)},
$$

provided that the denominator of the left-hand side is non-zero.

Proof.The proof is similar to the proof of Theorem 2.4 using Theorem 5.3 instead of Theorem 2.3 .

Let $L$ satisfy properties $L 1, L 2$ on a nonempty set $E$. Let $A$ be positive linear functional on $L$ with $A(1)=1$ and let $g \in L$. Then for a strictly monotone continuous function $\alpha$ such that $\alpha \circ g \in L$, the generalized quasi-arithmetic mean of the Mercer type, $\mathscr{M}_{\alpha}(g, A)$, of $g$ with respect to the positive linear functional $A$ and the function $\alpha$ is defined by (see [5])

$$
\mathscr{M}_{\alpha}(g, A)=\alpha^{-1}(\alpha(m)+\alpha(M)-A(\alpha \circ g)) .
$$

The following theorem holds. 
Theorem 5.5. Let $g \in L$ be such that the image of $g$ is a compact real interval $I=[m, M]$ and let $\alpha, \beta, \gamma: I \rightarrow \mathbb{R}$ be strictly monotone functions, $\alpha, \beta, \gamma \in C^{2}(I)$, such that $\alpha \circ g, \beta \circ g, \gamma \circ g,(\gamma \circ g)^{2} \in L$ and $(\gamma(m))^{2}+(\gamma(M))^{2}-A\left((\gamma \circ g)^{2}\right)-(\gamma(m)+\gamma(M)-A(\gamma \circ g))^{2} \neq 0$.

Then

$$
\frac{\alpha\left(\mathscr{M}_{\alpha}(g, A)\right)-\alpha\left(\mathscr{M}_{\gamma}(g, A)\right)}{\beta\left(\mathscr{M}_{\beta}(g, A)\right)-\beta\left(\mathscr{M}_{\gamma}(g, A)\right)}=\frac{\alpha^{\prime \prime}(\eta) \cdot \gamma^{\prime}(\eta)-\alpha^{\prime}(\eta) \cdot \gamma^{\prime \prime}(\eta)}{\beta^{\prime \prime}(\eta) \cdot \gamma^{\prime}(\eta)-\beta^{\prime}(\eta) \cdot \gamma^{\prime \prime}(\eta)}
$$

holds for some $\eta$ in the image of $g$, provided that the denominator of the left-hand side is nonzero.

Proof.The proof is similar to the proof of Theorem 3.1 using Theorem 5.4 instead of Theorem 2.4 with substitution

$$
\varphi=\alpha \circ \gamma^{-1}, \psi=\beta \circ \gamma^{-1}, g=\gamma \circ g, m=\gamma(m), M=\gamma(M) .
$$

Corollary 3. Let the conditions of Theorem 5.5 hold.

Let

$$
\chi(\eta)=\frac{\alpha^{\prime \prime}(\eta) \cdot \gamma^{\prime}(\eta)-\alpha^{\prime}(\eta) \cdot \gamma^{\prime \prime}(\eta)}{\beta^{\prime \prime}(\eta) \cdot \gamma^{\prime}(\eta)-\beta^{\prime}(\eta) \cdot \gamma^{\prime \prime}(\eta)}
$$

be invertible function.

Then

$$
\eta=\chi^{-1}\left(\frac{\alpha\left(\mathscr{M}_{\alpha}(g, A)\right)-\alpha\left(\mathscr{M}_{\gamma}(g, A)\right)}{\beta\left(\mathscr{M}_{\beta}(g, A)\right)-\beta\left(\mathscr{M}_{\gamma}(g, A)\right)}\right)
$$

is a mean, provided that the denominator of the term in the brackets is non-zero.

Proof. Since $\eta$ is in the image of $g$, it follows that

$$
\min _{t \in E} g(t) \leq \chi^{-1}\left(\frac{\alpha\left(\mathscr{M}_{\alpha}(g, A)\right)-\alpha\left(\mathscr{M}_{\gamma}(g, A)\right)}{\beta\left(\mathscr{M}_{\beta}(g, A)\right)-\beta\left(\mathscr{M}_{\gamma}(g, A)\right)}\right) \leq \max _{t \in E} g(t) .
$$

This shows that this is a mean.

Now, from the results given above, we can deduce corresponding results for the generalized power mean of the Mercer type, $Q_{r}(g, A)$, of $g$ with respect to the positive linear functional $A$ which is defined for $r \in \mathbb{R}$ by (see [9])

$$
Q_{r}(g, A)= \begin{cases}\left(m^{r}+M^{r}-A\left(g^{r}\right)\right)^{\frac{1}{r}}, & r \neq 0 \\ \frac{m M}{\exp (A(\log g))}, & r=0,\end{cases}
$$

where $g(t)>0$ for $t \in E$, $\log g \in L$ and $g^{r} \in L$ for $r \in \mathbb{R} \backslash\{0\}$. 
Corollary 4. Let $g \in L$ be such that the image of $g$ is a compact real interval $I=[m, M]$. Let $r, l, s \in \mathbb{R} \backslash\{0\}, r \neq l, s ; l \neq s$, such that $g^{r}, g^{l}, g^{s}, g^{2 s} \in L$ and $m^{2 s}+M^{2 s}-A\left(g^{2 s}\right)-\left(m^{s}+M^{s}-A\left(g^{s}\right)\right)^{2} \neq$ 0. Then

$$
\frac{Q_{r}^{r}(g, A)-Q_{s}^{r}(g, A)}{Q_{l}^{l}(g, A)-Q_{s}^{l}(g, A)}=\frac{r(r-s)}{l(l-s)} \cdot \eta^{r-l}
$$

holds for some $\eta$ in the image of $g$, provided that the denominator of the left-hand side is nonzero.

Proof. If we set

$$
\alpha(t)=t^{r}, \beta(t)=t^{l}, \gamma(t)=t^{s},
$$

in Theorem 5.5, we get the assertion (38).

Since $\eta$ is in the image of $g$, (38) suggests a new mean as it is

$$
\min _{t \in E} g(t) \leq\left(\frac{l(l-s)}{r(r-s)} \frac{Q_{r}^{r}(g, A)-Q_{s}^{r}(g, A)}{Q_{l}^{l}(g, A)-Q_{s}^{l}(g, A)}\right)^{\frac{1}{r-l}} \leq \max _{t \in E} g(t)
$$

for $r, l, s \in \mathbb{R} \backslash\{0\}, r \neq l, s ; l \neq s ; m^{2 s}+M^{2 s}-A\left(g^{2 s}\right)-\left(m^{s}+M^{s}-A\left(g^{s}\right)\right)^{2} \neq 0$.

From (38) it follows that we can define a new mean $\mathrm{M}_{r, l}^{[s]}(g, A)$ as follows

$$
\mathrm{M}_{r, l}^{[s]}(g, A)=\left(\frac{l(l-s)}{r(r-s)} \frac{Q_{r}^{r}(g, A)-Q_{s}^{r}(g, A)}{Q_{l}^{l}(g, A)-Q_{s}^{l}(g, A)}\right)^{\frac{1}{r-l}},
$$

for $r, l, s \in \mathbb{R} \backslash\{0\}, r \neq l, s ; l \neq s ; m^{2 s}+M^{2 s}-A\left(g^{2 s}\right)-\left(m^{s}+M^{s}-A\left(g^{s}\right)\right)^{2} \neq 0$.

Similarly, we can calculate some other cases for $r, l, s \in \mathbb{R}$ and we get the following definition of $\mathrm{M}_{r, l}^{[s]}(g, A)$.

Definition 5.6. Let $L$ satisfy properties $L 1, L 2$ on a nonempty set $E$ and let $A$ be a positive linear functional on $L$ with $A(1)=1$. Let $r, l, s \in \mathbb{R}$ and let $g \in L$ be such that the image of $g$ is a compact real interval $I=[m, M] \subset \mathbb{R}^{+}$, and $g^{r}, g^{l}, g^{s}, g^{2 s}, \log g, \log ^{2} g \in L$ for $r, l, s \in \mathbb{R} \backslash\{0\}$.

Then we define the Cauchy-type mean $\mathrm{M}_{r, l}^{[s]}(g, A)$ of $g$ with respect to the positive linear functional $A$ by

$$
\mathrm{M}_{r, l}^{[s]}(g, A)=\left(\frac{l(l-s)}{r(r-s)} \cdot \frac{Q_{r}^{r}(g, A)-Q_{s}^{r}(g, A)}{Q_{l}^{l}(g, A)-Q_{s}^{l}(g, A)}\right)^{\frac{1}{r-l}},
$$

for $r, l, s \neq 0 ; r \neq l, s ; l \neq s ; m^{2 s}+M^{2 s}-A\left(g^{2 s}\right)-\left(m^{s}+M^{s}-A\left(g^{s}\right)\right)^{2} \neq 0$;

$$
\mathrm{M}_{r, 0}^{[s]}(g, A)=\mathrm{M}_{0, r}^{[s]}(g, A)=\left(-\frac{s}{r(r-s)} \cdot \frac{Q_{r}^{r}(g, A)-Q_{s}^{r}(g, A)}{\log \left(Q_{0}(g, A)\right)-\log \left(Q_{s}(g, A)\right)}\right)^{\frac{1}{r}},
$$




$$
\begin{aligned}
& \text { for } r, s \neq 0 ; r \neq s ; m^{2 s}+M^{2 s}-A\left(g^{2 s}\right)-\left(m^{s}+M^{s}-A\left(g^{s}\right)\right)^{2} \neq 0 ; \\
& \qquad \mathrm{M}_{r, l}^{[0]}(g, A)=\left(\frac{l^{2}}{r^{2}} \cdot \frac{Q_{r}^{r}(g, A)-Q_{0}^{r}(g, A)}{Q_{l}^{l}(g, A)-Q_{0}^{l}(g, A)}\right)^{\frac{1}{r-l}},
\end{aligned}
$$

for $r, l \neq 0 ; r \neq l ; \log ^{2} m+\log ^{2} M-A\left(\log ^{2} g\right)-(\log m+\log M-A(\log g))^{2} \neq 0$;

where we suppose that all expressions are well defined.

Theorem 5.7. Let $a, b, c, d, s \in \mathbb{R}$ be such that $a \leq c, b \leq d, a \neq b, c \neq d$ and $s \neq a, b, c, d$.

Then, for $\mathrm{M}_{r, l}^{[s]}$ defined in Definition 5.6 we have that

$$
\mathrm{M}_{b, a}^{[s]} \leq \mathrm{M}_{d, c}^{[s]} .
$$

Proof. The proof is similar to the proof of Theorem 4.6, using adequate results from [2].

Remark 5.8. If we set the functional $A$ of the function $g$ to be

$$
A(g)=\int_{\Omega} g(u) d \mu(u)
$$

where $\Omega \subseteq \mathbb{R}^{n}$ is a convex set equipped with a probability measure $\mu$, we get the means of Cauchy-type given in [3] and also adequate results.

\subsection{Means of the Aczél type}

The following version of Jensen's inequality is valid too ([12, p.124-125]).

Theorem 5.9. Let $L$ satisfy properties $L 1, L 2$ on a nonempty set $E$ and let $A$ be a positive linear functional on $L$. Let $\varphi: I \rightarrow \mathbb{R}$ be a continuous convex function on an interval $I \subset \mathbb{R}$. Suppose that $w \in L$ with $w \geq 0$ on $E$ and $0<A(w)<u \in \mathbb{R}, \frac{u a-A(w g)}{u-A(w)} \in I(a \in I)$, where $g: E \rightarrow \mathbb{R}$ is such that $w g \in L$ and $w \varphi(g) \in L$. Then

$$
\varphi\left(\frac{u a-A(w g)}{u-A(w)}\right) \geq \frac{u \varphi(a)-A(w \varphi(g))}{u-A(w)} .
$$

Using this result we shall give two mean-value theorems for positive linear functionals.

Theorem 5.10. Let $L$ satisfy properties $L 1, L 2$ on a nonempty set $E$ and let $A$ be a positive linear functional on $L$. Let $\varphi: I \rightarrow \mathbb{R}, \varphi \in C^{2}(I)$, where $I \subset \mathbb{R}$ is a compact real interval. Suppose that $w \in L$ with $w \geq 0$ on $E$ and $0<A(w)<u \in \mathbb{R}, \frac{u a-A(w g)}{u-A(w)} \in I(a \in I)$, where $g: E \rightarrow \mathbb{R}$ is such that $w g, w g^{2}, w \varphi(g) \in L$. Then there exists some $\xi \in I$ such that the following holds

$$
\varphi\left(\frac{u a-A(w g)}{u-A(w)}\right)-\frac{u \varphi(a)-A(w \varphi(g))}{u-A(w)}=\frac{\varphi^{\prime \prime}(\xi)}{2}\left[\left(\frac{u a-A(w g)}{u-A(w)}\right)^{2}-\frac{u a^{2}-A\left(w g^{2}\right)}{u-A(w)}\right] .
$$


Proof. The proof is similar to the proof of Theorem 2.3 using Theorem 5.9 instead of Theorem 2.1 .

Theorem 5.11. Let $L$ satisfy properties $L 1, L 2$ on a nonempty set $E$ and let $A$ be a positive linear functional on $L$. Let $\varphi, \psi: I \rightarrow \mathbb{R}, \varphi, \psi \in C^{2}(I)$, where $I \subset \mathbb{R}$ is a compact real interval. Suppose that $w \in L$ with $w \geq 0$ on $E$ and $0<A(w)<u \in \mathbb{R}, \frac{u a-A(w g)}{u-A(w)} \in I(a \in I)$, where $g: E \rightarrow \mathbb{R}$ is such that $w g, w g^{2}, w \varphi(g), w \psi(g) \in L$ and $\left(\frac{u a-A(w g)}{u-A(w)}\right)^{2}-\frac{u a^{2}-A\left(w g^{2}\right)}{u-A(w)} \neq 0$. Then there exists some $\xi \in I$ such that the following holds

$$
\frac{\varphi\left(\frac{u a-A(w g)}{u-A(w)}\right)-\frac{u \varphi(a)-A(w \varphi(g))}{u-A(w)}}{\psi\left(\frac{u a-A(w g)}{u-A(w)}\right)-\frac{u \psi(a)-A(w \psi(g))}{u-A(w)}}=\frac{\varphi^{\prime \prime}(\xi)}{\psi^{\prime \prime}(\xi)},
$$

provided that the denominator of the left-hand side is non-zero.

Proof.The proof is similar to the proof of Theorem 2.4 using Theorem 5.10 instead of Theorem 2.3.

Let $L$ satisfy properties $L 1, L 2$ on a nonempty set $E$ and let $A$ be positive linear functional on $L$. Let $w \in L$ with $w \geq 0$ on $E, 0<A(w)<u \in \mathbb{R}$ and $a \in \mathbb{R}$. Let $g: E \rightarrow \mathbb{R}$ be such that $w g \in L$.

Then for a strictly monotone continuous function $\alpha: I \rightarrow \mathbb{R}(I \subseteq \mathbb{R}, a \in I)$ such that $w \alpha(g) \in L$, the generalized quasi-arithmetic mean of the Aczél type, $\mathfrak{M}_{\alpha}(g, A)$, of $g$ with respect to the positive linear functional $A$ and the function $\alpha$ is defined by

$$
\mathfrak{M}_{\alpha}(g, A)=\alpha^{-1}\left(\frac{u \alpha(a)-A(w \alpha(g))}{u-A(w)}\right) .
$$

The following theorem holds.

Theorem 5.12. Let $g: E \rightarrow \mathbb{R}$ be such that the image of $g$ is a compact real interval $I$ and let $\alpha, \beta, \gamma: I \rightarrow \mathbb{R}$ be strictly monotone functions, $\alpha, \beta, \gamma \in C^{2}(I)$, such that $w \alpha(g), w \beta(g), w \gamma(g), w(\gamma \circ$ $g)^{2} \in L$ and $\left(\frac{u \gamma(a)-A(w \gamma(g))}{u-A(w)}\right)^{2}-\frac{u(\gamma(a))^{2}-A\left(w(\gamma \circ g)^{2}\right)}{u-A(w)} \neq 0$.

Then

$$
\frac{\alpha\left(\mathfrak{M}_{\gamma}(g, A)\right)-\alpha\left(\mathfrak{M}_{\alpha}(g, A)\right)}{\beta\left(\mathfrak{M}_{\gamma}(g, A)\right)-\beta\left(\mathfrak{M}_{\beta}(g, A)\right)}=\frac{\alpha^{\prime \prime}(\eta) \cdot \gamma^{\prime}(\eta)-\alpha^{\prime}(\eta) \cdot \gamma^{\prime \prime}(\eta)}{\beta^{\prime \prime}(\eta) \cdot \gamma^{\prime}(\eta)-\beta^{\prime}(\eta) \cdot \gamma^{\prime \prime}(\eta)}
$$

holds for some $\eta$ in the image of $g$, provided that the denominator of the left-hand side is non-zero.

Proof.The proof is similar to the proof of Theorem 3.1 using Theorem 5.11 instead of Theorem 2.4 with substitution

$$
\varphi=\alpha \circ \gamma^{-1}, \psi=\beta \circ \gamma^{-1}, g=\gamma \circ g, a=\gamma(a)
$$


Corollary 5. Let the conditions of Theorem 5.12 hold.

Let

$$
\chi(\eta)=\frac{\alpha^{\prime \prime}(\eta) \cdot \gamma^{\prime}(\eta)-\alpha^{\prime}(\eta) \cdot \gamma^{\prime \prime}(\eta)}{\beta^{\prime \prime}(\eta) \cdot \gamma^{\prime}(\eta)-\beta^{\prime}(\eta) \cdot \gamma^{\prime \prime}(\eta)}
$$

be invertible function.

Then

$$
\eta=\chi^{-1}\left(\frac{\alpha\left(\mathfrak{M}_{\gamma}(g, A)\right)-\alpha\left(\mathfrak{M}_{\alpha}(g, A)\right)}{\beta\left(\mathfrak{M}_{\gamma}(g, A)\right)-\beta\left(\mathfrak{M}_{\beta}(g, A)\right)}\right)
$$

is a mean, provided that the denominator of the term in the brackets is non-zero.

Proof. Since $\eta$ is in the image of $g$, it follows that

$$
\min _{t \in E} g(t) \leq \chi^{-1}\left(\frac{\alpha\left(\mathfrak{M}_{\gamma}(g, A)\right)-\alpha\left(\mathfrak{M}_{\alpha}(g, A)\right)}{\beta\left(\mathfrak{M}_{\gamma}(g, A)\right)-\beta\left(\mathfrak{M}_{\beta}(g, A)\right)}\right) \leq \max _{t \in E} g(t) .
$$

This shows that this is a mean.

Now, from the results given above, we can deduce corresponding results for the generalized power mean of Aczél's type, $\mathscr{Q}_{r}(g, A)$, of $g$ with respect to the positive linear functional $A$, which is defined for $r \in \mathbb{R}$ by

$$
\mathscr{Q}_{r}(g, A)= \begin{cases}\left(\frac{u a^{r}-A\left(w g^{r}\right)}{u-A(w)}\right)^{\frac{1}{r}}, & r \neq 0 \\ \exp \left(\frac{u \log a-A(w \log g)}{u-A(w)}\right), & r=0,\end{cases}
$$

where $g(t)>0$ for $t \in E, w \log g \in L$ and $w g^{r} \in L$ for $r \in \mathbb{R} \backslash\{0\}$.

Corollary 6. Let $g: E \rightarrow \mathbb{R}$ be such that the image of $g$ is a compact real interval I. Let $r, l, s \in$ $\mathbb{R} \backslash\{0\}, r \neq l, s ; l \neq s$, such that $w g^{r}, w g^{l}, w g^{s}, w g^{2 s} \in L$ and $\left(\frac{u a^{s}-A\left(w g^{s}\right)}{u-A(w)}\right)^{2}-\frac{u a^{2 s}-A\left(w g^{2 s}\right)}{u-A(w)} \neq 0$. Then

$$
\frac{\mathscr{Q}_{s}^{r}(g, A)-\mathscr{Q}_{r}^{r}(g, A)}{\mathscr{Q}_{s}^{l}(g, A)-\mathscr{Q}_{l}^{l}(g, A)}=\frac{r(r-s)}{l(l-s)} \cdot \eta^{r-l}
$$

holds for some $\eta$ in the image of $g$, provided that the denominator of the left-hand side is nonzero.

Proof. If we set

$$
\alpha(t)=t^{r}, \beta(t)=t^{l}, \gamma(t)=t^{s},
$$


in Theorem 5.12, we get the assertion (47).

Since $\eta$ is in the image of $g$, (47) suggests a new mean as it is

$$
\min _{t \in E} g(t) \leq\left(\frac{l(l-s)}{r(r-s)} \frac{\mathscr{Q}_{r}^{r}(g, A)-\mathscr{Q}_{s}^{r}(g, A)}{\mathscr{Q}_{l}^{l}(g, A)-\mathscr{Q}_{s}^{l}(g, A)}\right)^{\frac{1}{r-l}} \leq \max _{t \in E} g(t)
$$

for $r, l, s \in \mathbb{R} \backslash\{0\}, r \neq l, s ; l \neq s ;\left(\frac{u a^{s}-A\left(w g^{s}\right)}{u-A(w)}\right)^{2}-\frac{u a^{2 s}-A\left(w g^{2 s}\right)}{u-A(w)} \neq 0$.

From (47) it follows that we can define a new mean $\mathrm{N}_{r, l}^{[s]}(g, A)$ as follows

$$
\mathrm{N}_{r, l}^{[s]}(g, A)=\left(\frac{l(l-s)}{r(r-s)} \frac{\mathscr{Q}_{s}^{r}(g, A)-\mathscr{Q}_{r}^{r}(g, A)}{\mathscr{Q}_{s}^{l}(g, A)-\mathscr{Q}_{l}^{l}(g, A)}\right)^{\frac{1}{r-l}},
$$

for $r, l, s \in \mathbb{R} \backslash\{0\}, r \neq l, s ; l \neq s ;\left(\frac{u a^{s}-A\left(w g^{s}\right)}{u-A(w)}\right)^{2}-\frac{u a^{2 s}-A\left(w g^{2 s}\right)}{u-A(w)} \neq 0$.

Similarly, we can calculate some other cases for $r, l, s \in \mathbb{R}$ and we get the following definition of $\mathrm{N}_{r, l}^{[s]}(g, A)$.

Definition 5.13. Let $L$ satisfy properties $L 1, L 2$ on a nonempty set $E$ and let $A$ be a positive linear functional on $L$. Let $w \in L$ with $w \geq 0$ on $E, 0<A(w)<u \in \mathbb{R}$ and $a \in \mathbb{R}$. Let $r, l, s \in \mathbb{R}$ and let $g: E \rightarrow \mathbb{R}$ be such that the image of $g$ is a compact real interval $I \subset \mathbb{R}^{+}(a \in I)$, and $w g, w g^{r}, w g^{l}, w g^{s}, w g^{2 s}, w \log g, w \log ^{2} g \in L$ for $r, l, s \in \mathbb{R} \backslash\{0\}$.

Then we define the Cauchy-type mean $\mathrm{N}_{r, l}^{[s]}(g, A)$ of $g$ with respect to the positive linear functional $A$ by

$$
\mathrm{N}_{r, l}^{[s]}(g, A)=\left(\frac{l(l-s)}{r(r-s)} \cdot \frac{\mathscr{Q}_{s}^{r}(g, A)-\mathscr{Q}_{r}^{r}(g, A)}{\mathscr{Q}_{s}^{l}(g, A)-\mathscr{Q}_{l}^{l}(g, A)}\right)^{\frac{1}{r-l}},
$$

for $r, l, s \neq 0 ; r \neq l, s ; l \neq s ;\left(\frac{u a^{s}-A\left(w g^{s}\right)}{u-A(w)}\right)^{2}-\frac{u a^{2 s}-A\left(w g^{2 s}\right)}{u-A(w)} \neq 0$.

$$
\mathrm{N}_{r, 0}^{[s]}(g, A)=\mathrm{N}_{0, r}^{[s]}(g, A)=\left(-\frac{s}{r(r-s)} \cdot \frac{\mathscr{Q}_{s}^{r}(g, A)-\mathscr{Q}_{r}^{r}(g, A)}{\log \left(\mathscr{Q}_{s}(g, A)\right)-\log \left(\mathscr{Q}_{0}(g, A)\right)}\right)^{\frac{1}{r}},
$$

for $r, s \neq 0 ; r \neq s ;\left(\frac{u a^{s}-A\left(w g^{s}\right)}{u-A(w)}\right)^{2}-\frac{u a^{2 s}-A\left(w g^{2 s}\right)}{u-A(w)} \neq 0$.

$$
\mathrm{N}_{r, l}^{[0]}(g, A)=\left(\frac{l^{2}}{r^{2}} \cdot \frac{\mathscr{Q}_{0}^{r}(g, A)-\mathscr{Q}_{r}^{r}(g, A)}{\mathscr{Q}_{0}^{l}(g, A)-\mathscr{Q}_{l}^{l}(g, A)}\right)^{\frac{1}{r-l}},
$$

for $r, l \neq 0 ; r \neq l ;\left(\frac{u \log a-A(w \log g)}{u-A(w)}\right)^{2}-\frac{u \log ^{2} a-A\left(w \log ^{2} g\right)}{u-A(w)} \neq 0$;

where we suppose that all expressions are well defined. 
Theorem 5.14. Let $a, b, c, d, s \in \mathbb{R}$ be such that $a \leq c, b \leq d, a \neq b, c \neq d$ and $s \neq a, b, c, d$.

Then, for $\mathrm{N}_{r, l}^{[s]}$ defined in Definition 5.13 we have that

$$
\mathrm{N}_{b, a}^{[s]} \leq \mathrm{N}_{d, c}^{[s]} .
$$

Proof. The proof is similar to the proof of Theorem 4.6, using adequate results from [2].

\subsection{Means of the Aczél type for discrete case}

Let $x=\left(x_{1}, \ldots, x_{n}\right) \in I^{n} \subset \mathbb{R}^{n}$ and $p=\left(p_{1}, \ldots, p_{n}\right)$ be the real $n$-tuples such that

$$
p_{1}>0, p_{2}, \ldots, p_{n} \leq 0, P_{n}>0
$$

where $P_{n}=\sum_{i=1}^{n} p_{i}$ and $\frac{1}{P_{n}} \sum_{i=1}^{n} p_{i} x_{i} \in I$.

Setting in Theorem 5.9 that $E=\{2,3, \ldots, n\}, u=p_{1}, a=x_{1}, w(i)=-p_{i}$ and $g(i)=x_{i}$ for $i \in E, A(w)=\sum_{i \in E} w(i)$, then for continuous convex function $f: I \rightarrow \mathbb{R}$ we get the reverse Jensen inequality

$$
f\left(\frac{1}{P_{n}} \sum_{i=1}^{n} p_{i} x_{i}\right) \geq \frac{1}{P_{n}} \sum_{i=1}^{n} p_{i} f\left(x_{i}\right) .
$$

Now we can define similar results as in the previous subsection, and also the new means of the Cauchy type. Firstly, let us define the adequate power means.

Definition 5.15. Let $r \in \mathbb{R}$ and let $x \in \mathbb{R}_{+}^{n}$ and $p$ be the real $n$-tuples such that

$$
p_{1}>0, p_{2}, \ldots, p_{n} \leq 0, P_{n}>0
$$

where $\sum_{i=1}^{n} p_{i} x_{i}^{r}>0$ for $r \in \mathbb{R} \backslash\{0\}$.

Then the power means of order $r \in \mathbb{R}$ are defined by

$$
\bar{M}_{r}(x, p)= \begin{cases}\left(\frac{1}{P_{n}} \sum_{i=1}^{n} p_{i} x_{i}^{r}\right)^{\frac{1}{r}}, & r \neq 0 ; \\ \left(\prod_{i=1}^{n} x_{i}^{p_{i}}\right)^{\frac{1}{P_{n}}}, & r=0 .\end{cases}
$$

The definition of the means of the Cauchy type follows.

Definition 5.16. Let $r, l, s \in \mathbb{R}$. Let $x=\left(x_{1}, \ldots, x_{n}\right)$ be a positive real $n$ - tuple such that not all $x_{i}$ are equal and let $p$ be a real $n$-tuple such that

$$
p_{1}>0, p_{2}, \ldots, p_{n} \leq 0, P_{n}>0,
$$

where $\sum_{i=1}^{n} p_{i} x_{i}^{r}, \sum_{i=1}^{n} p_{i} x_{i}^{l}, \sum_{i=1}^{n} p_{i} x_{i}^{s}>0$ for $r, l, s \in \mathbb{R} \backslash\{0\}$. 
Then we define the Cauchy-type mean $\mathbf{M}_{r, l}^{[s]}(x, p)$ in which we suppose that all expressions are well defined.

$$
\begin{aligned}
& \mathbf{M}_{r, l}^{[s]}(x, p)=\left(\frac{l(l-s)}{r(r-s)} \frac{\bar{M}_{r}^{r}(x, p)-\bar{M}_{s}^{r}(x, p)}{\bar{M}_{l}^{l}(x, p)-\bar{M}_{s}^{l}(x, p)}\right)^{\frac{1}{r-l}}, \quad r, l, s \neq 0 ; r \neq l, s ; l \neq s ; \\
& \mathbf{M}_{r, 0}^{[s]}(x, p)=\mathbf{M}_{0, r}^{[s]}(x, p)=\left(\frac{s\left[\bar{M}_{r}^{r}(x, p)-\bar{M}_{s}^{r}(x, p)\right]}{r(r-s)\left[\log \bar{M}_{s}(x, p)-\log \bar{M}_{0}(x, p)\right]}\right)^{\frac{1}{r}}, \quad \quad r, s \neq 0 ; r \neq s ; \\
& \mathbf{M}_{s, l}^{[s]}(x, p)=\mathbf{M}_{l, s}^{[s]}(x, p)=\left(\frac{l(l-s)}{s} \frac{\sum_{i=1}^{n} p_{i} x_{i}^{s} \log x_{i}-\bar{M}_{s}^{s}(x, p) \log \bar{M}_{s}(x, p)}{\bar{M}_{l}^{l}(x, p)-\bar{M}_{s}^{l}(x, p)}\right)^{\frac{1}{s-l}}, l, s \neq 0 ; l \neq s ; \\
& \mathbf{M}_{s, 0}^{[s]}(x, p)=\mathbf{M}_{0, s}^{[s]}(x, p)=\left(\frac{\sum_{i=1}^{n} p_{i} x_{i}^{s} \log x_{i}-\bar{M}_{s}^{s}(x, p) \log \bar{M}_{s}(x, p)}{\log \bar{M}_{s}(x, p)-\log \bar{M}_{0}(x, p)}\right)^{\frac{1}{s}}, \quad s \neq 0 \\
& \mathbf{M}_{r, l}^{[0]}(x, p)=\left(\frac{l^{2}\left(\bar{M}_{r}^{r}(x, p)-\bar{M}_{0}^{r}(x, p)\right)}{r^{2}\left(\bar{M}_{l}^{l}(x, p)-\bar{M}_{0}^{l}(x, p)\right)}\right)^{\frac{1}{r-l}}, \quad \quad l, r \neq 0 ; r \neq l \\
& \mathbf{M}_{r, 0}^{[0]}(x, p)=\mathbf{M}_{0, r}^{[0]}(x, p)=\left(\frac{2\left[\bar{M}_{r}^{r}(x, p)-\bar{M}_{0}^{r}(x, p)\right]}{r^{2}\left[\bar{M}_{2}^{2}(\log x, p)-\bar{M}_{1}^{2}(\log x, p)\right]}\right)^{\frac{1}{r}}, \quad r \neq 0 . \\
& \mathbf{M}_{r, r}^{[s]}(x, p)=\exp \left(-\frac{2 r-s}{r(r-s)}+\frac{\sum_{i=1}^{n} p_{i} x_{i}^{r} \log x_{i}-\bar{M}_{s}^{r}(x, p) \log \bar{M}_{s}(x, p)}{\bar{M}_{r}^{r}(x, p)-\bar{M}_{s}^{r}(x, p)}\right), \quad r, s \neq 0 ; r \neq s ; \\
& \mathbf{M}_{r, r}^{[0]}(x, p)=\exp \left(-\frac{2}{r}+\frac{\sum_{i=1}^{n} p_{i} x_{i}^{r} \log x_{i}-\bar{M}_{0}^{r}(x, p) \log \bar{M}_{0}(x, p)}{\bar{M}_{r}^{r}(x, p)-\bar{M}_{0}^{r}(x, p)}\right), \quad r \neq 0 ; \\
& \mathbf{M}_{0,0}^{[0]}(x, p)=\exp \left(\frac{1}{3} \frac{\sum_{i=1}^{n} p_{i}\left(\log x_{i}\right)^{3}-\left(\log \bar{M}_{0}(x, p)\right)^{3}}{\sum_{i=1}^{n} p_{i}\left(\log x_{i}\right)^{2}-\left(\log \bar{M}_{0}(x, p)\right)^{2}}\right) \\
& \mathbf{M}_{s, s}^{[s]}(x, p)=\exp \left(-\frac{1}{s}+\frac{\sum_{i=1}^{n} p_{i} x_{i}^{s}\left(\log x_{i}\right)^{2}-\bar{M}_{s}^{s}(x, p)\left(\log \bar{M}_{s}(x, p)\right)^{2}}{2\left(\sum_{i=1}^{n} p_{i} x_{i}^{s} \log x_{i}-\left(\bar{M}_{s}^{s}(x, p) \log \bar{M}_{s}(x, p)\right)\right)}\right), \quad s \neq 0 \\
& \mathbf{M}_{0,0}^{[s]}(x, p)=\exp \left(\frac{1}{s}+\frac{\sum_{i=1}^{n} p_{i}\left(\log x_{i}\right)^{2}-\left(\log \bar{M}_{s}(x, p)\right)^{2}}{\left.2\left(\sum_{i=1}^{n} p_{i} \log x_{i}-\log \bar{M}_{s}(x, p)\right)\right)}\right), \quad s \neq 0
\end{aligned}
$$

Theorem 5.17. Let $a, b, c, d, s \in \mathbb{R}$ be such that $a \leq c, b \leq d$. Then, for $\mathbf{M}_{r, l}^{[s]}$ defined in Definition 5.16 we have that

$$
\mathrm{M}_{b, a}^{[s]} \leq \mathrm{M}_{d, c}^{[s]}
$$

Proof. The proof is similar to the proof of Theorem 4.6. 


\section{References}

[1] H. Alzer, Eine Doppelungleichung für Integrale, Anzeiger Öster Akad. Wiss. Math-naturwiss. Klasse, 127 (1990), 37-40.

[2] M. Anwar and J. Pečarić, On logarithmic convexity for differences of power means and related results, Math. Inequal. Appl., 12(2009), 81-90.

[3] M. Anwar and J. Pečarić, Cauchy Means of Mercer's type, Utilitas Mathematica, 84(2011), 201-208.

[4] M. Anwar, J. Pečarić, New means of Cauchy's type, J. Inequal. Appl., 2008 (2008), Article ID 163202, 10 pages

[5] W. S. Cheung, A. Matković, and J. Pečarić, A variant of Jessen inequality and generalized means, JIPAM, J. Inequal. Pure Appl. Math. 7(2006), Article 10.

[6] B. Jessen, Bemaerkinger om konvekse Funktioner og Uligheder imellem Middelvaerdier I., Mat. Tidsskrift, B, (1931), 17-28.

[7] A. McD. Mercer, An "error term" for the Ky Fan inequality, J. Math. Anal. Appl., 220 (1998), 774-777.

[8] A. McD. Mercer, Some new inequalities involving elementary mean values, J. Math. Anal. Appl., 229 (1999), 677-681.

[9] A. McD. Mercer, A monotonicity property of power means, JIPAM, J. Inequal. Pure Appl. Math. 3(2002), Article 40.

[10] D. S. Mitrinović, J. E. Pečarić, A. M. Fink, Classical and new Inequalities in Analysis, Kluwer Academic Publishers, Dordrecht/Boston/London, 1993.

[11] J. E. Pečarić, I. Perić and H. M. Srivastava, A family of the Cauchy type mean-value theorems, J. Math. Anal. Appl., 306 (2005), 730-739.

[12] J. E. Pečarić, F. Proschan and Y. L. Tong, Convex functions, partial orderings, and statistical applications, Mathematics in Science and Engineering, vol. 187, Academic Press, 1992.

[13] J. E. Pečarić, M. Rodić Lipanović and H. M. Srivastava, Some mean-value theorems of the Cauchy type, Fract. Calc. Appl. Anal. 9(2006), 143-158.

${ }^{1}$ Center for Advanced Mathematics and Physics, National University of Sciences and Technology, Islamabad, Pakistan.

E-mail: matloob_t@yahoo.com

${ }^{2}$ Faculty of Textile Technology, University of Zagreb, Prilaz baruna Filipovića 28a, 10000 Zagreb, Croatia. Abdus Salam School of Mathematical Sciences, GC University, Lahore, Pakistan.

E-mail: pecaric@mahazu.hazu.hr

${ }^{3}$ Faculty of Textile Technology, University of Zagreb, Prilaz baruna Filipovića 28a, 10000 Zagreb, Croatia.

E-mail:mrodic@ttf.hr 